\title{
Arrhythmia Mechanism in Acute Hyperglycemic Conditions
}

\author{
Achmad Shofwan Hadi ${ }^{a}$, Soebagijo Adi ${ }^{b}$, Hanestya Oky Hermawan ${ }^{\text {a }}$, Primasitha \\ Maharany Harsoyo ${ }^{\mathrm{a}}$ \\ ${ }^{\mathrm{a}}$ kardiounair@gmail.com \\ ${ }^{a}$ Cardiology and Vascular Medicine Department, Faculty of Medicine of Airlangga University, Surabaya, 60286, Indonesia



\begin{abstract}
Acute hyperglycemia or stress hyperglycemia commonly occurs in critically ill patients and could be a marker of disease severity. Hyperglycemia at hospital admission is commonly found in patients with cardiovascular disease, regardless of their diabetic status; one of them is arrhythmia. Arrhythmia or dysrhythmia is a heart rhythm disorder that refers to any disturbance in the frequency, regularity, location of origin or electrical impulses conduction of the heart. Arrhythmias associated with stress hyperglycemia include atrial arrhythmias, supraventricular tachycardia (SVT), high-grade (atrioventricular) AV block, and ventricular arrhythmias. Type 2 diabetes was also associated with a significantly increased risk of arrhythmias and sudden death. Hyperglycemia plays an important role in arrhythmia through various mechanisms. In this review, we describe the correlation and mechanism by which arrhythmias may occur in hyperglycemic conditions
\end{abstract}

Keywords: Arrhytmia; Acute hyperglicemic: Cardiovascular disease; Sudden death

\section{Introduction}

Acute hyperglycemia or stress hyperglycemia commonly occurs in critically ill patients and could be a marker of disease severity. In addition, glucose levels, both at initial hospital admission and mean glucose level during hospitalization, are strongly associated with patient outcomes (Marik et al., 2013). In 1878, Claude Bernard, a French physiologist, described hyperglycemia occurrence during hemorrhagic shock, and it is now known that associated acute illness or injury might cause hyperglycemia, insulin resistance, and glucose intolerance, collectively termed as stress hyperglycemia (Badawi et al., 2012). Hyperglycemia at hospital admission is commonly found in patients with coronary heart disease (CHD), regardless of their diabetic status. Martalena et al. stated that the prevalence of hyperglycemia at initial hospital admission in CHD patients at intensive care unit was $43.43 \%$. Furthermore, arrhythmia is a common complication in CHD (Fariz et al., 2015).

Arrhythmia or dysrhythmia is a heart rhythm disorder that refers to any disturbance in the frequency, regularity, location of origin or electrical impulses conduction of the heart (Thaler, 2013). Arrhythmias associated with stress hyperglycemia include atrial arrhythmias, supraventricular tachycardia (SVT), highgrade (atrioventricular) AV block, and ventricular arrhythmias. Arrhythmia prevalence in hospitals is still unclear. However, epidemiological data obtained from the New England Medical Journal (2001) stated that structural abnormalities of the coronary arteries were the cause of $80 \%$ of heart rhythm disorders that might lead to sudden death. Meanwhile, Agarwal et al., in their study in India in 2017, stated that type 2 diabetes was associated with a significantly increased risk of arrhythmias and sudden death. This was described in their study involving 100 patients with type 2 diabetes mellitus and arrhythmias for two years in India. As a result of the study, 62\% of the patients had prolonged QTc associated with cardiac autonomous neuropathy and a high arrhythmia incidence (Agarwal et al., 2017). 


\section{Stress Hyperglycemia Pathogenesis}

The primary role of metabolic response to stress is to increase substrate flux, especially glucose, to the tissues that are dependent on it. The brain is the main glucose consumer in fasting state and its utilization rate is insulin independent. Therefore, central glucose delivery maintenance depends only on plasma glucose concentration and adequate blood flow to the brain. Thus, stress hyperglycemia could be viewed as a means of ensuring adequate glucose delivery to the brain during bodily stress and prolonged fasting conditions.

During a condition in which cerebral blood flow is normal, glucose uptake into the central nervous system (CNS) is sufficient if plasma glucose level is above $70 \mathrm{mg} / \mathrm{dL}$. Increasing the body's production of ketone from the liver during bodily stress or prolonged fasting conditions and their utilization by the brain might reduce the obligatory need for carbohydrates by approximately $50 \%$ without impairing nerve function. When the supply to the brain is reduced, it compromises the functioning of the brain's glucose uptake and ultimately results in neuronal death. Hence, blood flow compromise to the brain during bodily stress or prolonged fasting conditions leads to stress hyperglycemia to compensate for the decrease of glucose delivery to the brain (Moses, 2013).

\section{Acute Disease, Stress Response, and Stress Hyperglycemia}

The stress response is mediated largely by the hypothalamic-pituitary-adrenal (HPA) axis and the sympathoadrenal system. In general, there is a gradation in gradual stress response. Cortisol and catecholamine levels were correlated with surgery type, disease severity, Glasgow Coma Scale, and APACHE score (Marik et al., 2013). Under severe stress conditions, the cortisol output from adrenal gland increases 10fold (about $300 \mathrm{mg}$ hydrocortisone per day). In patients with shock, epinephrine plasma concentration is increased 50-fold and norepinephrine level is increased 10-fold. The increased release of these stress hormones induces a variety of metabolic, cardiovascular, and immune effects aimed at restoring homeostasis during stress. Furthermore, the HPA axis, the sympathoadrenal system, and pro-inflammatory cytokines (TNF- $\alpha$, IL-1 and IL-6) act collectively and synergistically to induce stress hyperglycemia (Marik et al., 2013).

The neuroendocrine response to stress is characterized by excessive gluconeogenesis, glycogenolysis, and insulin resistance. Stress hyperglycemia is primarily caused by increased liver glucose output compared to impaired glucose extraction by cells in tissues. The metabolic effects of cortisol include increasing blood glucose concentrations through key enzymes activation involved in hepatic gluconeogenesis and glucose uptake inhibition in peripheral tissues such as skeletal muscle (Marik et al., 2013). Both epinephrine and norepinephrine stimulate liver gluconeogenesis and glycogenolysis; norepinephrine has the additional effect of increasing glycerol supply to the liver via lipolysis. Inflammatory mediators, in particular cytokines such as TNF-, IL-1, IL-6, and C-reactive protein, also induce peripheral insulin resistance (Figure 2). In addition, changes in adipokines (increased zinc-alpha glycoprotein and decreased adiponectin) of adipose tissue during acute disease are thought to play a key role in insulin resistance development (Olsson et al., 2009).

\section{Hyperglycemia and Cardiovascular Disease}

Hyperglycemia has been shown to play a significant role in endothelial dysfunction process. Postprandial hyperglycemia is significantly correlated with postprandial myocardial perfusion (Chenscarabelli et al., 2006), and it might exert a deleterious effect on myocardial vasodilator function (Srinivasan et al., 1998). Hyperglycemia causes several changes in vascular tissue that accelerate atherosclerosis incidence. Several mechanisms are thought to explain this event, including non-enzymatic glycosylation of proteins and lipids, protein kinase C (PKC) activation, and oxidative stress (Colin et al., 2007). These processes in their courses will lead to the increase of intracellular free radicals (reactive oxygen species) 
formation, loss of collagen elasticity and flexibility, reduced NO production resulting in smooth muscle contraction, and atherosclerosis process acceleration, which in turn will cause a series of atherosclerotic clinical events and damages on end-organs (Aritonang et al., 2013).

\section{Arrhythmia Pathophysiology}

Arrhythmia is a condition in which the heart beats in an abnormal rhythm. Arrhythmias could be a primary, isolated disease such as supraventricular tachycardia (SVT), Wolf-Parkinson-White syndrome, lone atrial fibrillation, atrial flutter, fascicular ventricular tachycardia, idiopathic right ventricular outflow tract (RVOT) tachycardia, long QT syndrome, and Brugada syndrome, or secondary due to certain diseases such as mitral stenosis, coronary heart disease, or induced by drugs. When an arrhythmia occurs, the heart could beat irregularly, too rapidly (tachycardia), too slowly (bradycardia), or not beating at all (asystole) (Lilly et al., 2016). Tachycardia is further characterized as supraventricular when it involves the atrium or atrioventricular (AV) node and as ventricular when it originated in the His-Purkinje system or ventricle. Heart rhythm disturbance results from changes in impulse formation, impulse conduction, or both. Arrhythmia's presentation ranges from benign general palpitations to severe symptoms such as low cardiac output resulting in cardiogenic shock, heart failure, and death. Arrhythmia could occur at any age (Lilly et al., 2016).

As explained in cardiac pathophysiology lecture, electrical impulse formation of the heart arises from the intrinsic automaticity of specialized cardiac cells. Automaticity refers to the cell's ability to spontaneously depolarize to a threshold voltage to generate an action potential. Although atrial and ventricular myocytes do not have this property under normal conditions, cells of the specialized conduction system have natural automaticity and are therefore called pacemaker cells. The specialized conduction systems include the sinoatrial node (SA-node), the AV node region, and the ventricular conduction system. The ventricular conduction system consists of the His bundle, left and right bundle branches, and Purkinje fibers. In pathological situations, myocardial cells outside the conduction system could also acquire automaticity (Lilly et al., 2016).

In general, arrhythmias occur due to changes in impulse formation and conduction. Both might manifest as tachyarrhythmia or bradyarrhythmia (Figure 3). In tachyarrhythmias, the causes are automaticity, triggered activity, and reentrant. Automaticity arises when an accelerated phase 4 action potential occurs. This type of arrhythmia is triggered by acute and critical conditions such as acute myocardial infarction, electrolyte disturbances, acidosis and includes stress hyperglycemia.

The most common cause of arrhythmias is reentrant, which is usually caused by chronic processes such as scarred myocardium and cardiomyopathy. The presence of healthy and diseased myocardial tissue in close proximity is ideal for reentrants. Furthermore, a rare arrhythmia mechanism is triggered activity, which is a combination of automaticity and a reentrant process (Yamin et al., 2012).

\section{Mechanism of Arrhythmias in Hyperglycemic Conditions}

Sanjuan et al.. (2011) revealed that hyperglycemia on admission was associated with an increased risk of ventricular arrhythmias during hospitalization. Koracevic et al.. (2008) reported that atrial fibrillation (AF) was more common in the hyperglycemic group on admission compared to the non-hyperglycemic group. In Indonesia, Fariz et al.. (2015) at Cipto Mangunkusumo Hospital Jakarta investigated the correlation between hyperglycemia during admission with arrhythmia incidence during treatment in patients with acute coronary syndrome (ACS). The conclusion of this study was hyperglycemia during admission might increase arrhythmia risk during treatment in ACS patients.

The underlying mechanism could be explained in several ways. First, insulin resistance and acute hyperglycemia increase free fatty acid concentrations and predispose to sympathetic-vagal nerve imbalance (Smit et al., 2006). Lipid accumulation in the myocardium causes calcium overload, while sympathetic-vagal 
nerve imbalance causes a prolonged QT interval. Second, insulin resistance and inflammatory hyperglycemia are increased in ACS, contributing to structural and electrical remodeling (Wang et al., 2015). Third, hyperglycemia increases free radical production and decreases nitric oxide (NO) production, resulting in prolonged myocardial repolarization, tachycardia potentiation, delayed protection from ventricular arrhythmias, and sympathetic modulation of ventricular electrophysiological properties. Fourth, hyperglycemia activates the intracrine renin-angiotensin system, which results in permeability disturbance of the gap junction. This leads to myocardial dysfunction and arrhythmias. Fifth, hyperglycemia increases platelet aggregation, which might result in changes in action potentials and myocardial contractility, and action potential spread (Fariz et al., 2015).

\section{Effects of Hyperglycemia on Reduced Nitric Oxide (NO) Bioavailability in Arrhythmia Process}

It has been described that hyperglycemia might induce oxidative stress and lead to accumulation of advanced glycation end-products, both of which lead to NO elimination through reduced NO bioavailability. This is due to vascular effect reversal induced during hyperglycemia by L-arginine, thereby reducing NO bioavailability in the process (Giugliano et al., 1997). This process of reduced NO bioavailability might partly explain the increased incidence of ventricular arrhythmias in patients with poor glycemic control. Moreover, the modulating effect of NO on arrhythmia development is described in these processes: suppression of arrhythmia-induced-ouabain (Na-K-ATP-ase inhibitor) by NO derived from endothelial activation of myocyte nitric oxide synthase (NOS), potentiation of tachycardia through NOS inhibition, cardioprotection inhibition against ventricular arrhythmias through NOS formation, and sympathetic effect modulation on ventricular electrophysiological properties, and possibly through reduced NO-induced norepinephrine release in the heart (to reduce ventricular arrhythmia incidence). However, it should be noted that cardiomyocyte overexpression of NOS has been shown to result in impaired atrial and/or atrioventricular node conduction, manifesting as bradyarrhythmia, heart block, and sudden cardiac death in rats (Mungrue et al., 2002).

\section{Diabetic Hyperglycemia Activates $\mathrm{Ca}^{2+}$-Calmodulin-dependent Protein Kinase II (CaMKII) and Arrhythmia via O-linked Glycosylation}

$\mathrm{Ca}^{2+}$-Calmodulin-dependent protein kinase II (CaMKII) is a regulatory node in the heart and brain, and its chronic activation could be a pathological process. CaMKII activation seen in heart failure could directly induce pathological changes in ion channels, $\mathrm{Ca}^{2+}$ handling, and gene transcription (Anderson et al., 2011). O-Linked -N-acetylglucosamine (O-GlcNAc) is an intracellular carbohydrate that dynamically converts serine and threonine amino acids through their hydroxyl groups on nuclear and cytoplasmic proteins. O-GlcNAc modifies a myriad of nucleocytoplasmic proteins and is involved in transcription, ubiquitination, cell cycle, and response to stress (Butkinaree et al., 2009).

Acute hyperglycemia triggers covalent modification of CaMKII by O-linked N-acetylglucosamine (O-GlcNAc). O-GlcNAc modification of CaMKII activates CaMKII autonomously, creating a molecular memory even after $\mathrm{Ca}^{2+}$ level has decreased. CaMKII-modified O-GlcNAc is elevated in the heart and brain of diabetic humans and mice. In cardiomyocytes, elevated glucose significantly increases CaMKII-dependent activation of spontaneous sarcoplasmic reticulum (SR) $\mathrm{Ca}^{2+}$ events that might contribute to cardiac mechanical dysfunction and arrhythmias. This effect is prevented by pharmacological inhibition of O-GlcNAc signaling or CaMKII $\delta$ genetic ablation. In an intact and adequately perfused heart, arrhythmia is enhanced by increased glucose via the O-GlcNAc- and CaMKII-dependent pathways. In diabetic animals, acute blockade of O-GlcNAc inhibits arrhythmogenesis. Thus, the O-GlcNAc modification of CaMKII is a novel signaling event in a pathway that might critically contribute to cardiac and neurologic pathophysiology in diabetes and other diseases (Erickson et al., 2013). 


\section{Conclusion}

Acute hyperglycemia or stress hyperglycemia is one of response mechanisms to acute illness, injury, or trauma of the body, through neurohormonal processes that are regulated by the central nervous system. Cardiovascular diseases such as acute myocardial infarction and acute heart failure are some of the clinical forms that might induce stress hyperglycemia. On the other hand, hyperglycemia plays an important role in cardiovascular disease through various mechanisms such as endothelial dysfunction, increased formation of intracellular free radicals (reactive oxygen species), loss of collagen elasticity and flexibility, reduced NO production resulting in smooth muscle contraction, and accelerated atherosclerosis.

Arrhythmia is an important manifestation of cardiovascular disease that could be independent (primary) or a complication of the underlying disease (secondary). Arrhythmia presentation ranges from benign general palpitations to severe symptoms such as low cardiac output resulting in cardiogenic shock, heart failure, and death. Arrhythmias are known to have a correlation with hyperglycemia incidence, especially stress hyperglycemia. There are several mechanisms underlying arrhythmia occurrence in hyperglycemic conditions. The most important mechanism is through reduced NO bioavailability and activation of Ca2+-Calmodulin-dependent Protein Kinase II (CaMKII) and arrhythmias through O-linked Glycosylation. Several studies have revealed the occurrence of arrhythmic events in hyperglycemic conditions, especially acute hyperglycemia or hyperglycemia at the time of patient admission to the hospital, and revealed a correlation between them. Several studies reported arrhythmia occurrence in acute hyperglycemia, mainly acute hyperglycemia and hyperglycemia during hospital admission, and revealed the correlation between these two variables. Most studies reported that the mechanism of arrhythmia occurrences in hyperglycemia is associated with preexisting heart conditions in patients. There are still no studies that reported arrhythmia occurrence that is primarily due to hyperglycemia in both diabetic and non-diabetic patients. Thus, there is still room for further studies to reveal the correlation between arrhythmias and diabetes mellitus, and larger, multicenter randomized controlled trials are needed to formulate more structured management of arrhythmias that occur in acute hyperglycemia.

\section{Acknowledgements}

We thank the head of department and head of study program Cardiology and Vascular Medicine, Faculty of Medicine Airlangga University, Surabaya, Indonesia

\section{References}

Agarwal G, Singh SK. Arrhythmias in type 2 diabetes mellitus. Indian J Endocr Metab 2017;21:715-8.

Aritonang FA, Soemantri D. Penyakit Jantung Koroner dengan Diabetes Mellitus. Penyakit Jantung Koroner Manajemen Komprehensif: Seri Buku Ilmiah Departemen Kardiologi dan Kedokteran Vaskular FK Unair RSUD Dr. Soetomo. 2013. p.180-181.

Badawi O, Waite MD, Fuhrman SA, Zuckerman IH: Association between intensive care unit-acquired dysglycemia and in-hospital mortality. Crit Care Med 2012, 40:3180-3188.

Chen-scarabelli C, Scarabelli MT. (2006). Suboptimal Glycemic Control, Independently of QT Interval Duration, Is Associated with Increased Risk of Ventricular Arrhythmias in a High-Risk Population. Pacing and Clinical Electrophysiology 2006;29(1):9-14. Source: Medscape

Colin B, Jean-Claude T, Martial GB. Coronary heart disease in patients with diabetes. JACC. 2007; 49 (6): $631-642$.

Erickson JR, Pereira L, Wang L, Han G, Ferguson A, Dao K, Copeland RJ, Despa F, Hart GW, Ripplinger CM, Bers DM. (2013). Diabetic Hyperglycemia activates CaMKII and Arrhythmias by O linked Glycosylation. Nature. 2013 October 17; 502(7471): 372376. doi:10.1038/nature12537.

Fariz AMZZ, Nasution SA, Purnamasari D, Mansjoer A. (2015). The Influence of Hyperglycemia at Admission on In-hospital Arrhythmia Patients with Acute Coronary Syndrome. Acta Medica Indonesiana-The Indonesian Journal of Internal Medicine. 47: 291-296.

Jernas M, Olsson B, Sjoholm K, Sjogren A, Rudemo M, Nellgard B, Carlsson LM, Sjostrom CD: Changes in adipose tissue gene 
expression and plasma levels of adipokines and acute-phase proteins in patients with critical illness. Metabolism. 2009, 58:102108

Koracevic GP, Petrovic S, Damjanovic M, Stanojlovic T. Association of stress hyperglycemia and atrial fibrillation in myocardial infarction. Wien Klin Wochenschr. 2008;120(13-14):409-13.

Marik PE, Bellomo R: Stress hyperglycemia: an essential survival response! Critical Care 2013, 17:305.

Moses, A. (2013) Stress Hyperglycemia. Medicine Update 2013, Mumbai: APIINDIA, p.178-180.

Prust, MJ, Stevenson WG, Strichartz GR, Leonard S. Lilly. (2003). Mechanism of Cardiac Arrhytmia. Pathophysiology of heart disease : a collaborative project of medical students and faculty. Philadelphia :Lippincott Williams \& Wilkins, p.287-290

Sanjuan R, Blasco ML, Maicas HM, et al. Acute myocardial infarction: high risk ventricular tachyarrhythmias and admission glucose level in patients with and without diabetes mellitus. Curr Diabetes Rev. 2011;7(2):126-34.

Smit JWA, Romijn JA. Acute insulin resistance in myocardial ischemia: causes and consequences. Seminars in Cardiothoracic and Vascular Anesthesia. 2006;10(3):215-9.

Srinivasan M, Herrero P, McGill JB, Bennik J, Heere B, Lesniak D, Davila-Roman VG, Gropler RJ. The effects of plasma insulin and glucose on myocardial blood flow in patients with type 1 diabetes mellitus. J Am Coll Cardiol 2005; 46(1):42-48.

Wang J, Yang M, Zhu J. Mechanisms of new-onset atrial fibrillation complicating acute coronary syndrome. Herz. 2015;40(Suppl 1):1826.

Yamin M, Rilantono L, (2012). Takikardia Ventrikular Apa Yang Harus Diwaspadai ?. Penyakit Kardiovaskular 5 Rahasia. Jakarta: Badan Penerbit FKUI. p.411. 